\title{
User Identification and Key Regeneration System Employing Rotated Reference Images of the Iris
}

\author{
Guilherme Nunes Melo, Valdemar C. da Rocha Jr. e José S. Lemos-Neto
}

\begin{abstract}
A new test called rotation search is proposed for user identification and cryptographic key regeneration in systems employing a digital representation of the iris which is called the iris code. When applied to the BIOSECURE, CASIA and NISTICE data bases the rotation search shows, on average, a two fold reduction in false rejection ratio (FRR) with a false acceptance ratio (FAR) equal to zero, in comparison with the standard search employed in other systems. The highest improvement reached in FRR by the rotation search against the standard search is about 100 times for a single iris and 85 times for the two irides of a user, and in many cases the measured FRR is equal to zero.
\end{abstract}

Index Terms-Iris code, biometry, coding, authentication.

\section{INTRODUCTION}

$\mathbf{U}$ SER identification employing biometric data is now a reality in many computer based systems including banking, voting, access to vaults, etc. Applications using biometrics will certainly grow and will reach more users as soon as they become more reliable, by granting access to genuine users and denying access to impostors with high probability. Many identification systems using biometrics operate on user fingerprints, the palm, the face or the iris. In this paper we restrict attention to systems employing digital iris data (iris code). The reason for this choice is due to the fact that the iris code presents the highest entropy in comparison to other biometric data currently in use [2]. Our idea is to take advantage of the higher iris code entropy in order to achieve higher security levels against impostors. We refer to the test proposed in this paper as rotation search, which performs a search to identify a user by employing both, rotated reference images and rotated test images. As described in the sequel, rotation search applied to the BIOSECURE, CASIA and NISTICE data bases shows, on average, a two fold reduction in false rejection ratio (FRR) with a false acceptance ratio (FAR) equal to zero, in comparison with the standard search employed in other systems [3]-[5]. The highest improvement reached in FRR by the rotation search against the standard search is about 100 times for a single iris and 85 times for both irides, and in many cases the measured FRR is equal to zero.

The Associate Editors coordinating the review of this manuscript and approving it for publication were Prof. Cecilio José Lins Pimentel and Prof. Marcelo da Silva Pinho.

The authors are with the Communications Research Group, Department of Electronics and Systems, Federal University of Pernambuco, 50740-550, Recife, PE, Brazil, Emails: \{guilherme.nmelo, vcr, jose.lemosnt\}@ufpe.br.

A preliminary version of this paper was presented in XXXIII Simpósio Brasileiro de Telecomunicações (SBrT'15), Juiz de Fora, MG, Brazil, September 1-4, 2015 [1].

Digital Object Identifier (DOI): 10.14209/jcis.2016.5.
Recent research in iris biometric features has focused mainly on finding new methods to obtain irises codes [6][9]. Nevertheless, security aspects of biometric systems have also been subject of recent interest [10]-[13]. In this paper our main focus is on the performance aspects of potentially practical biometric systems which have received relatively little attention so far.

The rest of this paper is organized as follows. In Section II we describe the main relevant aspects of the data bases employed. In Section III we present the key regeneration system proposed in this paper which employs error-correcting codes. In Section IV we describe the new proposed test. In Section V the experiments using the proposed system and the proposed test are presented and the results are compared to the results of the system in [4] and [5]. Finally, in Section VI, we present our conclusions.

\section{DAtA BASES}

Typically, data bases contain sets of images for each user, and that includes both reference images $\left(I_{\text {ref }}\right)$ and test images $\left(I_{\text {sam }}\right)$. A reference image is understood to be generated under ideal conditions while a test image is understood to be generated by a user identification equipment, i.e., in less than ideal conditions. Following [3], for each image in a data base, a binary string of length 1,188 bits is derived from an infrared image of an iris. These binary strings are denominated iris codes. The iris codes obtained from reference images and test images are respectively denoted by $\theta_{\text {ref }}$ and $\theta_{\text {sam }}$. In this paper, the iris codes used in the tests are derived from the following data bases: BIOSECURE [14], CASIA [14] and NIST-ICE [15].

The BIOSECURE and CASIA data bases are formed by 1, 200 images each, originating from 60 distinct users, each user having 20 images, where 10 images are reference images and the remaining 10 images are test images. Another possible interpretation is to consider 30 distinct users, being 10 reference images for the right eye, 10 test images for the right eye, 10 reference images for the left eye, and 10 test images for the left eye. Using these two data bases, 6,000 tests for genuine users are possible in each data base by using one iris image at a time, or 3,000 tests are possible by using iris images for both eyes at the same time. The NIST-ICE data base is formed by 2,953 images, which are divided in two tests namely, ICE-exp1 and ICE-exp2. The ICE-exp1 test is formed by 124 users having a total of 1,425 images and refers to the right eye, while the ICE-exp2 test is formed by 120 users having a total of 1,528 images and refers to the left eye. The ICE-exp1 data base allows 12,214 tests for genuine 
users while the ICE-exp2 test allows 14, 653 tests. In the NISTICE data base the number of images per user is not fix, being possible to find users having a number of images ranging from 1 to a maximum of 31 images.

When working with both irides using the NIST-ICE data base, we check for each user the number $N_{L}$ of images for his left iris and the number $N_{R}$ of images for his right iris. We then choose the smallest number, i.e., we choose $\min \left\{N_{L}, N_{R}\right\}$, to perform our experiments. For example, if a user has $N_{L}=11$ and $N_{R}=7$, we consider 7 images for each iris. This is the same rule used by [4], and in this manner we can compare our results with those reported in [4]. We are then able to perform 6,229 tests in a single experiment using both irides for the NIST-ICE data base. Consequently a control file is required in order to keep a list of which images will be used in each test. We call regular data bases those data bases which have the same number of images per user, e.g., BIOSECURE and CASIA, otherwise we call them irregular data bases, e.g., NIST-ICE.

\section{Key REgeneration System PROPOSED}

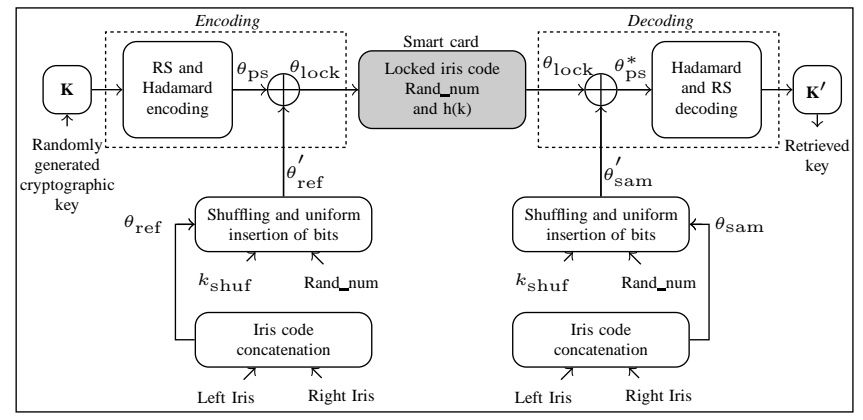

Fig. 1. Key regeneration uni-biometric or multi-biometric system, employing smart card, Left iris and/or Right iris and password.

Figure 1 presents a block diagram for the key regeneration system proposed in this paper. We use this system for testing images available from the iris data bases of Section II. The proposed system is essentially that proposed in [3] except for the insertion of random numbers [4] instead of zeros and for the block labeled "Iris code concatenation", which performs concatenation of irides' codes in experiments employing both irides. When a single iris is employed for an experiment, the "Iris code concatenation" block just passes forward its input to its output. The main features of the proposed system are described in the sequel.

As illustrated in Figure 1, encoding of the cryptographic key $\mathbf{K}$ is performed sequentially by employing first a ReedSolomon (RS) code [16, p. 294] and then a binary Hadamard code $\left(2^{k}, k+1,2^{k-1}\right)$ [16, p. 44]. Following [3], for the case where a single iris is employed we consider shortened RS codes of block length 61 over $\operatorname{GF}\left(2^{6}\right)$ and the Hadamard $(32,6,16)$ code. For the case where both irides are employed, we consider shortened RS codes of block length 61 over $\mathrm{GF}\left(2^{7}\right)$ and the Hadamard $(64,7,32)$ code, the same used in [4]. The error-correcting capability $t_{\mathrm{RS}}$ of the RS code is adjusted for the range $1 \leq t_{\mathrm{RS}} \leq 22$ satisfying the relation $k=61-2 t_{\mathrm{RS}}$, where $k$ denotes the number of information symbols of the RS code. Values of $t_{\mathrm{RS}}$ greater than 22 are avoided because they increase the FAR, i.e., for $t_{\mathrm{RS}}>22$ the scheme begins to erroneously consider some impostors as genuine users.

The shuffling operation for a single iris, according to [3], consists in segmenting the 1,188 bit iris code into 198 blocks of 6 bits each, and these blocks are then reordered using a randomly generated binary shuffling key, $k_{\text {shuf }}$, of length 198 bits as follows. The block reordering, i.e., the shuffling operation, can be more clearly described by making an analogy with the situation which occurs when passengers are going to board a plane. Originally the 198 blocks, i.e., passengers, form a single queue and sequentially each block (passenger) in the queue is assigned a token which is either a 1 or a 0 , obtained by sequentially reading the bits in the shuffling key. Then, respecting the original order of the blocks in the queue, two new queues are formed. One queue receives those blocks for which their token is 1 (premium class passengers) and the other queue receives those blocks for which their token is 0 (standard class passengers). Obeying their arrival order, blocks with token 1 board first, followed by blocks with token 0 , i.e., the shuffled sequence contains the blocks with token 1 followed by the blocks with token 0 . When both irides are employed, the shuffing and uniform insertion of bits operation is the same as described earlier, except for two details: first, $2 \times 1,188=2,376$ bits are employed, which result from the concatenation of the left iris code and the right iris code, and second, $k_{\text {shuf }}=396$ bits is employed to maintain 6 bits as the length of each block in the shuffling operation.

The uniform insertion of bits consists of concatenating a cascade of blocks formed by three bits from the iris code followed by two bits from a random or pseudo random sequence. Since the iris code consists of a binary sequence of length 1, 188 bits, it follows that after bit insertions a sequence of length 1,980 bits results. However, since the block length of $\theta_{\mathrm{ps}}$ is $61 \times 32=1,952$, it is necessary to delete 28 bits from the sequence of length 1,980 and thus achieve a length of 1,952 for $\theta_{\text {ref }}^{\prime}$. Among the 28 bits deleted there are 18 bits from the iris code which are lost. The lost iris code bits correspond to approximately $1.52 \%$ of the total, and it was verified that the error correction performance is not significantly affected by this loss. When both irides are employed, the iris code is a binary sequence of length 2,376 bits, and after bit insertions a sequence of length 3,960 bits results. However, for the Hadamard code with $k=6$, the block length of $\theta_{\mathrm{ps}}$ is $61 \times 64=3,904$, and it is necessary to delete 56 bits from the sequence of length 3,960 and thus achieve a length of 3,904 for $\theta_{\text {ref }}^{\prime}$. Among the 56 bits deleted there are 34 bits of the iris code which are lost. The lost iris code bits correspond to approximately $1.43 \%$ of the total, and again it was verified that the error correction performance is not significantly affected by this loss.

Decoding starts with the Hadamard code, which means that for each one of its codewords a 6 bit byte is delivered to form one symbol of a codeword for the RS code for a single iris. When both irides are used, the decoder for the $(64,7,32)$ Hadamard code delivers a 7 bit byte to form one symbol of a 


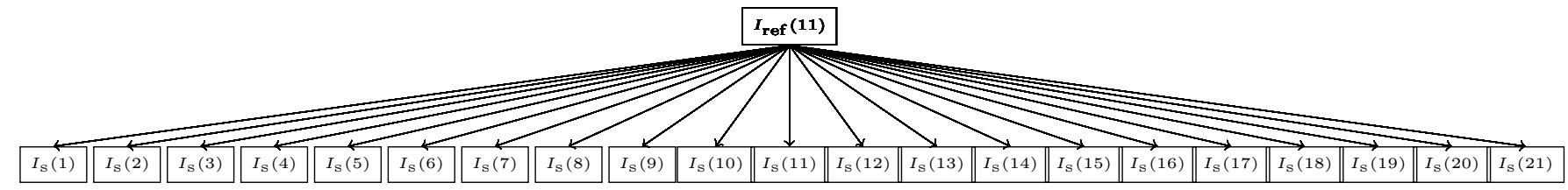

Fig. 2. Standard search technique, where $I_{\mathrm{S}}(r)=I_{\mathrm{sam}}(r), 1 \leq r \leq 21$.

codeword of the RS code. A little trick [3] which speeds up the computer simulation is then employed. Since we know the RS codeword that has been generated, we compare this generated RS codeword with the word coming out of the Hadamard decoder and count the number of symbol errors. The number of symbol errors $t$ is compared against the number of errors $t_{\mathrm{RS}}$ that the RS code can correct. If $t \leq t_{\mathrm{RS}}$ then we recover the cryptographic key $\mathbf{K}$, thus avoiding the actual decoding of the $\mathrm{RS}$ code and reducing processing time.

\section{New Proposed Test}

In this section we propose a new user identification test called rotation search. Before we present the proposed test, we briefly describe the test used in [3]-[5], that we call standard search, in order to compare it to the rotation search test. We emphasize that the tests use the iris codes $\theta_{\text {ref }}$ and $\theta_{\text {sam }}$ instead of their respective $I_{\text {ref }}$ and $I_{\text {sam }}$, as indicated in the block diagram in Figure 1.

\section{A. Standard search}

In order to allow testing a given key regeneration system based on iris both $I_{\text {ref }}$ and $I_{\text {sam }}$ are used for each user. Furthermore, for each stored image, either a reference image or a test image, the data base stores 20 rotated versions of that image, i.e., a total of 21 images. We denote by $I_{\text {ref }}(r, i, u)$ the $r^{\text {th }}$ rotated version of reference image number $i$, belonging to user $u$, for $1 \leq r \leq 21,1 \leq i \leq N$, and $1 \leq u \leq U$. Similarly, we write $I_{\text {sam }}(r, j, u), 1 \leq j \leq M$ to number test images. We refer to each comparison of images as a test, and call the set of tests for all users an experiment.

For the systems in [3]-[5], the tests are performed for each user $u$ by picking one reference image for $r=11$, i.e. $I_{\text {ref }}(11, i, u)$, and comparing it with up to 21 versions of a corresponding test image $I_{\text {sam }}(r, j, u), 1 \leq r \leq 21$. If a positive identification occurs when testing image $I_{\text {sam }}(r, j, u)$, then the test with image $j$ stops with match acceptance. However, if no positive identification is reached for $1 \leq r \leq 21$, then an identification error for test image $j$ is computed, and if $j<M$ then the test image $I_{\text {sam }}(r, j+1, u)$ is the next one to be compared with $I_{\text {ref }}(11, i, u)$. When the value $j=M$ is reached, the tests with image $I_{\text {ref }}(11, i, u)$ are concluded, reference image $I_{\text {ref }}(11, i+1, u)$ is then selected in order to continue the tests, which then proceed in a manner similar to what was done for reference image $i$. When all reference images for user $u$ have been selected, i.e., when $i=N$ and there are no more test images for user $u$, i.e., when $j=M$, tests with user $u$ are concluded, user $u+1$ is then selected, together with the corresponding reference and test images in order to continue the tests. The experiment finishes when the tests with user $u=U$ are completed. Hereafter, we refer to this procedure as standard search which is illustrated in Figure 2.

We remark that in the standard search up to 21 rotations of each test image $I_{\text {sam }}(r, j, u), 1 \leq r \leq 21,1 \leq j \leq M$, are performed per reference image $I_{\mathrm{ref}}(11, i, u), 1 \leq i \leq N$, and no rotation of reference images are employed. Summarizing, in the standard search 21 rotations are performed for each one of $M$ test images, for $N$ reference images and for $U$ users.

\section{B. Rotation search}

The rotation search performs a search to identify a user by employing both, rotated reference images $I_{\text {ref }}(r, i, u)$ and rotated test images $I_{\mathrm{sam}}(r, j, u)$. A sketch of the test performed by the rotation search is described next. Figure 3 illustrates, for a randomly selected user $u^{*}$, a few tests employing reference image $i, 1 \leq r \leq 21$, i.e., $I_{\text {ref }}\left(r, i, u^{*}\right)$, for $r \in\{1,6,11,16,21\}$. In general, up to 441 tests for each test image can be performed to verify authenticity, by employing up to 21 distinct rotated versions of each reference image and up to 21 distinct rotated versions of each test image. Clearly many more situations are considered in a rotation search in comparison with a standard search and as a consequence there is an increase in the required processing time. We remark that each one of the data bases considered already in Section II contains all the rotated images required by the rotation search to perform the tests, i.e., no new data was necessary or was required by the proposed system.

The rotation search consists in systematically comparing a pair of images where one of them is either a reference image or one of its rotated versions $I_{\text {ref }}(r, i, u)$, and the other image in the pair is either a test image or one of its rotated versions $I_{\text {sam }}(r, j, u)$, where $1 \leq r \leq 21,1 \leq i \leq N, 1 \leq j \leq M$ and $1 \leq u \leq U$. For example, for the BIOSECURE or CASIA data bases, using a single iris an experiment employs $N=10$ reference images per user, $M=10$ test images per user and $U=60$ distinct users. Thus a total of $10 \times 10 \times 60=6,000$ tests are performed, and for each test a worst case maximum of $r \times r=21 \times 21=441$ verifications are performed when all rotated versions of both reference images and test images are required. In this manner the simulation time for rotation search is increased when rotated versions of a reference image need to be used. When using both irides, with the BIOSECURE or CASIA data bases, an experiment employs $N=10$ reference images per user, $M=10$ test images per user and $U=30$ distinct users. Thus a total of $10 \times 10 \times 30=3,000$ tests are performed, and for each test also a worst case maximum of 441 verifications are performed when all rotated versions of both reference images and test images are required. 


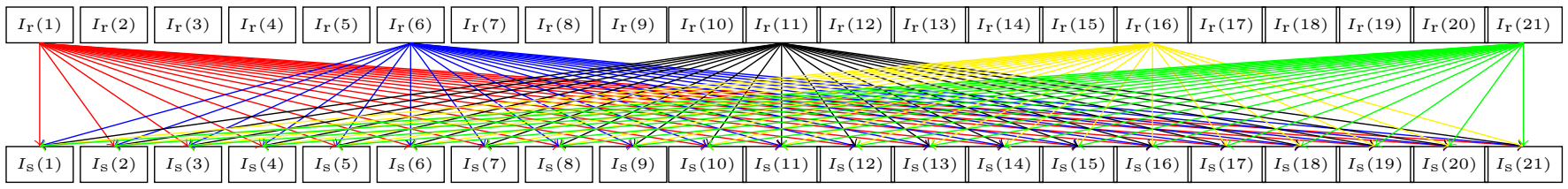

Fig. 3. Example, where $I_{\mathrm{r}}(r)=I_{\text {ref }}(r), 1 \leq r \leq 21, I_{\mathrm{S}}(r)=I_{\mathrm{sam}}(r), 1 \leq r \leq 21$, for $I_{\text {ref }}(r): r=1 ; r=6 ; r=11 ; r=16 ; r=21$, in our proposed rotation search.

\section{EXPERIMENTS AND RESULTS}

In this section we compare the standard search and the rotation search in terms of their implementation efficiency. Furthermore, we analyze the performance of the proposed key regeneration system of Section III in terms of the values of FAR and FRR, and compare them to the results in [4] and [5]. The simulations were performed in a computer equipped with an Intel core $i 7$ third generation processor, 128GB SSD HD, $8 \mathrm{~GB}$ of RAM, which was used for both $\mathrm{C}++$ and MATLAB implementation and comparison.

\section{A. Implementation efficiency}

This paper is a follow up of the research reported in [3]-[5] with new contributions. The software employed in [3] and [4] was developed using the proprietary programming language MATLAB, which comes with a series of pre-programmed mathematical operations and routines. For the development and implementation of the tests proposed here we noticed that the use of MATLAB was not the best choice as its data processing speed could not meet the speeds required. Consequently, the $\mathrm{C}++$ programming language was employed because of the faster responses and reduction in the time to perform each test. The data in the available data bases is in the form of MATLAB compact tables, which do not allow a fast access by a software developed outside of MATLAB. For this reason, the iris codes in the data bases had to be converted to a new format which allows a more efficient access using $\mathrm{C}++$. In this manner, 21 files were converted for each data base plus three control files for the NIST-ICE data base. The control files define which images belong to a certain user and who these users are.

The implementation efficiency of the rotation search is assessed by counting the number of comparisons per second and comparing it against the standard search method. We notice that the processing time is directly proportional to the number of decoding failures, which in turn grows with increasingly poor quality of user iris images. For any reference image or rotated reference image, $I_{\text {ref }}\left(r_{1}, i, u\right)$, the search procedure is halted as soon as a positive identification is found with a rotated test image $I_{\text {ref }}\left(r_{2}, j, u\right)$, or if all possible comparisons have been tried without success. The rotation search then selects another rotated version of the same reference image $I_{\text {ref }}\left(r_{1}^{\prime}, i, u\right)$ in order to perform comparison with rotated test images. After all rotated versions of a given reference image have been tested and there was no positive identification then an error is declared. The rotation system then selects another reference image to continue the search. When performing tests with the rotation search, it was observed that the least number of identification errors occurred for the data base NIST-ICEexp1, followed by the data bases CASIA, BIOSECURE and NIST-ICE-exp2. The BIOSECURE is a regular data base which presents the worst results for the rotation search among regular data bases. For this reason, we have chosen the BIOSECURE data base for measuring implementation efficiency. The reason for not choosing the data base NIST-ICE-exp2 is because it is an irregular data base.

For a single iris, the results obtained using the standard search are presented in Table I, which shows the time in seconds necessary to perform the tests as well as the number of comparisons for 60 users, with 10 reference images and 10 test images, for $1 \leq t_{\mathrm{RS}} \leq 22$. We considered the average time taken over three repetitions of each test. Observing the data in Table I we notice that the standard search runs roughly 20 times faster when implemented in $\mathrm{C}++$ than its MATLAB implementation.

TABLE I

STANDARD SEARCH SIMULATION RESULTS FOR THE BIOSECURE DATA BASE USING MATLAB AND C++, FOR A SINGLE IRIS.

\begin{tabular}{|c|c|c|}
\hline & MATLAB & $\mathrm{C}++$ \\
\hline Time (s) & 12,295 & 598 \\
\hline Comparisons & 132,000 & 132,000 \\
\hline Comparisons/s & 11 & 220 \\
\hline
\end{tabular}

The way we implemented the software for rotation search allows access to all data in intermediary stages of a test. In this manner we can identify which user images show more errors and subsequently identify which users were harder to be identified. We observed in the tests performed that most of the positive identifications occurred when we used $I_{\text {ref }}(r, i, u)=$ $I_{\text {ref }}(11, i, u)$ reference images and compared them with more central test images like $I_{\text {sam }}(10, j, u), I_{\text {sam }}(11, j, u)$ and $I_{\text {sam }}(12, j, u)$, not necessarily in this order. We took into consideration this observation in our implementation of the rotation search, by testing central images first and, if necessary, continue the test using images farther from the center $(r=11)$.

TABLE II

COMPARATIVE RESULTS USING THE BIOSECURE DATA BASE, FOR A SINGLE IRIS, (*) DENOTES SIMULATION EMPLOYING CENTRALIZED SEARCH.

\begin{tabular}{|c|c|c|c|}
\hline & $\begin{array}{c}\text { Standard } \\
(\mathrm{MATLAB})\end{array}$ & $\begin{array}{c}\text { Standard* } \\
(\mathrm{C}++)\end{array}$ & $\begin{array}{c}\text { Rotation* } \\
(\mathrm{C}++)\end{array}$ \\
\hline Time $(\mathrm{s})$ & 12,295 & 253 & 1,936 \\
\hline Comparisons & 132,000 & 132,000 & 132,000 \\
\hline Comparisons/s & 11 & 521 & 68 \\
\hline
\end{tabular}


TABLE III

PERCENT FRR FOR THE STANDARD, KANADE ET AL. [5] AND ROTATION SEARCH TEST FOR A SINGLE IRIS. FAR IS ALWAYS ZERO FOR THE ROTATION SEARCH TEST.

\begin{tabular}{|c|c|c|c|c|c|c|c|c|c|c|c|c|}
\hline & \multicolumn{3}{|c|}{ BIOSECURE V1 } & \multicolumn{3}{|c|}{ CASIA V2 } & \multicolumn{3}{|c|}{ ICE-exp1 } & \multicolumn{3}{|c|}{ ICE-exp2 } \\
\hline$t_{\mathrm{RS}}$ & Standard & Kanade & Rotation & Standard & Kanade & Rotation & Standard & Kanade & Rotation & Standard & Kanade & Rotation \\
\hline 1 & 30.79 & 30.53 & 15.15 & 50.19 & 49.70 & 23.11 & 48.79 & 49.39 & 21.37 & 52.01 & 52.99 & 24.34 \\
\hline 2 & 22.15 & 22.12 & 11.04 & 36.10 & 35.78 & 15.09 & 34.30 & 33.26 & 13.75 & 37.37 & 37.74 & 16.17 \\
\hline 3 & 16.52 & 16.37 & 8.63 & 26.08 & 26.27 & 10.82 & 23.97 & 24.26 & 9.53 & 27.12 & 25.78 & 11.44 \\
\hline 4 & 13.12 & 12.88 & 7.49 & 18.99 & 19.25 & 7.81 & 17.12 & 16.50 & 6.97 & 19.95 & 20.10 & 8.73 \\
\hline 5 & 10.75 & 10.65 & 6.63 & 14.55 & 14.82 & 5.85 & 12.60 & 12.67 & 5.23 & 15.10 & 16.25 & 6.59 \\
\hline 6 & 9.32 & 8.98 & 5.99 & 11.46 & 11.70 & 4.42 & 9.34 & 10.31 & 3.83 & 11.65 & 11.81 & 4.89 \\
\hline 7 & 8.34 & 8.35 & 5.39 & 9.13 & 9.52 & 3.29 & 7.13 & 7.29 & 2.79 & 9.27 & 9.42 & 3.69 \\
\hline 9 & 6.71 & 6.60 & 4.06 & 5.79 & 5.97 & 1.69 & 4.27 & 4.61 & 1.56 & 5.73 & 6.26 & 1.93 \\
\hline 10 & 6.08 & 5.87 & 3.40 & 4.65 & 4.85 & 1.09 & 3.32 & 3.63 & 1.16 & 4.42 & 4.54 & 1.38 \\
\hline 11 & 5.35 & 5.28 & 2.74 & 3.75 & 3.77 & 0.64 & 2.48 & 2.48 & 0.83 & 3.40 & 3.49 & 0.91 \\
\hline 12 & 4.68 & 4.57 & 2.00 & 2.83 & 3.13 & 0.28 & 1.82 & 2.13 & 0.62 & 2.57 & 3.05 & 0.63 \\
\hline 13 & 4.04 & 3.97 & 1.59 & 2.13 & 2.12 & 0.14 & 1.38 & 1.46 & 0.39 & 1.88 & 2.12 & 0.46 \\
\hline 14 & 3.25 & 3.25 & 0.95 & 1.49 & 1.57 & 0.03 & 1.06 & 1.04 & 0.24 & 1.41 & 1.41 & 0.35 \\
\hline 15 & 2.54 & 2.67 & 0.60 & 1.00 & 1.07 & 0.01 & 0.80 & 0.76 & 0.16 & 1.08 & 1.09 & 0.26 \\
\hline 16 & 1.98 & 2.00 & 0.36 & 0.60 & 0.63 & 0.00 & 0.58 & 0.69 & 0.12 & 0.77 & 0.94 & 0.18 \\
\hline 18 & 0.97 & 1.00 & 0.06 & 0.20 & 0.25 & 0.00 & 0.29 & 0.38 & 0.05 & 0.39 & 0.46 & 0.08 \\
\hline 19 & 0.61 & 0.63 & 0.04 & 0.12 & 0.15 & 0.00 & 0.22 & 0.26 & 0.04 & 0.31 & 0.39 & $\overline{0.03}$ \\
\hline 20 & 0.27 & 0.42 & 0.00 & 0.05 & 0.05 & 0.00 & 0.16 & 0.15 & 0.03 & 0.25 & 0.29 & 0.01 \\
\hline 21 & 0.17 & 0.23 & 0.00 & 0.03 & 0.03 & 0.00 & 0.11 & 0.13 & 0.02 & 0.19 & 0.20 & 0.01 \\
\hline 22 & 0.07 & 0.13 & 0.00 & 0.01 & 0.00 & 0.00 & 0.08 & 0.11 & 0.01 & 0.13 & 0.13 & 0.01 \\
\hline
\end{tabular}

As a result we produced Table II, where it is seen that the standard search became about 47 times faster then its MATLAB implementation. Still referring to Table II, we observe that rotation search using $\mathrm{C}++$ is about six times faster than the standard search using MATLAB. By analyzing test data we estimate that the rotation search would take approximately 4,500 seconds if a centralized search is not employed, still 2.7 times faster than the standard search using MATLAB.

The centralized search, i.e., a rotation search which moves from central images to more peripheral images, obeys the following search sequence order: $I_{\mathrm{sam}}(11, j, u), I_{\mathrm{sam}}(11-l, j, u)$, $I_{\text {sam }}(11+l, j, u), 1 \leq l \leq 10$. The same search sequence order employed in $I_{\text {sam }}(r, j, u)$ is employed in reference images $I_{\text {ref }}(r, i, u), 1 \leq r \leq 21$. This change in the search order reduced the image identification processing time by a factor greater than two. On the other hand, the standard search implemented in MATLAB selects test images $I_{\text {sam }}(r, j, u)$, $1 \leq r \leq 21$, in increasing order, beginning with $I_{\text {sam }}(1, j, u)$ and ending with $I_{\text {sam }}(21, j, u)$.

\section{B. Performance of the proposed system for a single iris}

Table III presents three columns for each data base. The first column of each data base contains the result obtained with our $\mathrm{C}++$ software, running the standard search as implemented in [4], and serves as a reference for comparison between our results and those obtained in [5]. The second column of each data base contains the results obtained in [5], and the third column of each data base contains the results obtained with our new proposed rotation search.

Table III shows that in all tests performed, for various values of $t_{\mathrm{RS}}$ and for all data bases considered, the rotation search consistently shows better results than the current search procedure. For $t_{\mathrm{RS}}=15$ in CASIA data base, our proposed scheme achieved a result about 100 times better than that obtained in [5]. We emphasize that for all values of $t_{\mathrm{RS}}$, FAR is always zero using the rotation search test while for the system in [5], FAR is greater than zero for $t_{\mathrm{RS}} \geq 10$.

Figure 4 shows a bar diagram illustration of the data in Table III. The bar labelled standard search represents the arithmetic mean of all results of \%FRR, considering all data bases, for each $t_{\mathrm{RS}}$.

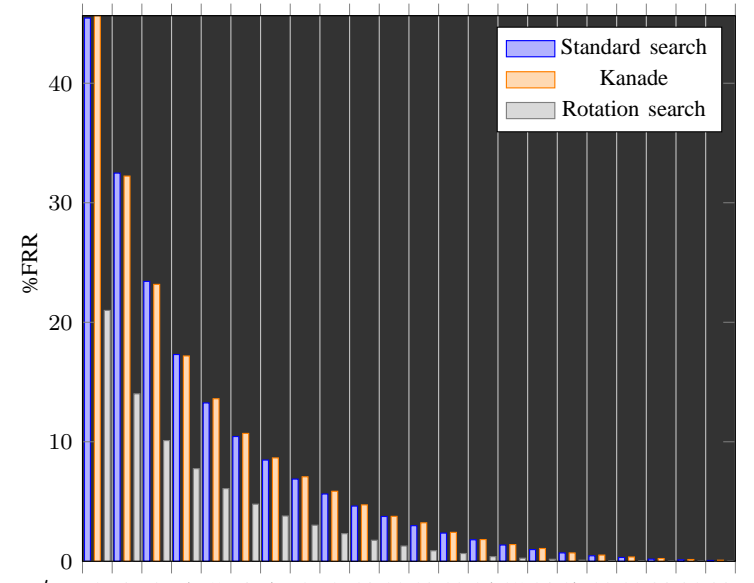

Fig. 4. Bar diagram illustrating percent average FRR in Table III versus $t_{R S}$, for standard search, Kanade [5] and rotation search.

The bar labelled Kanade [5] represents the arithmetic mean of all results of \%FRR, considering all data bases, for each $t_{\mathrm{RS}}$. Finally, the bar labelled rotation search represents the arithmetic mean of all results of \%FRR, considering all data 
bases, for each $t_{\mathrm{RS}}$. When $t_{\mathrm{RS}}$ is increased, \%FRR decreases, and in some cases it is zero or almost zero, so its corresponding bar in Figure 4 practically vanishes.

Figure 5 illustrates the situation where no user separation technique is employed and Figure 6 illustrates the user separation resulting from the application of shuffling key $\left(k_{\text {shuf }}\right)$, random numbers and rotation search. We notice that Figure 6 is very similar to the one obtained in [4], which means that the inclusion of a shuffling key in our scheme did not significantly contribute for user separation. It should be emphasized that the significant reduction in FRR obtained here, in comparison with the system in [5], is due essentially to the rotation search in combination with error-correction.

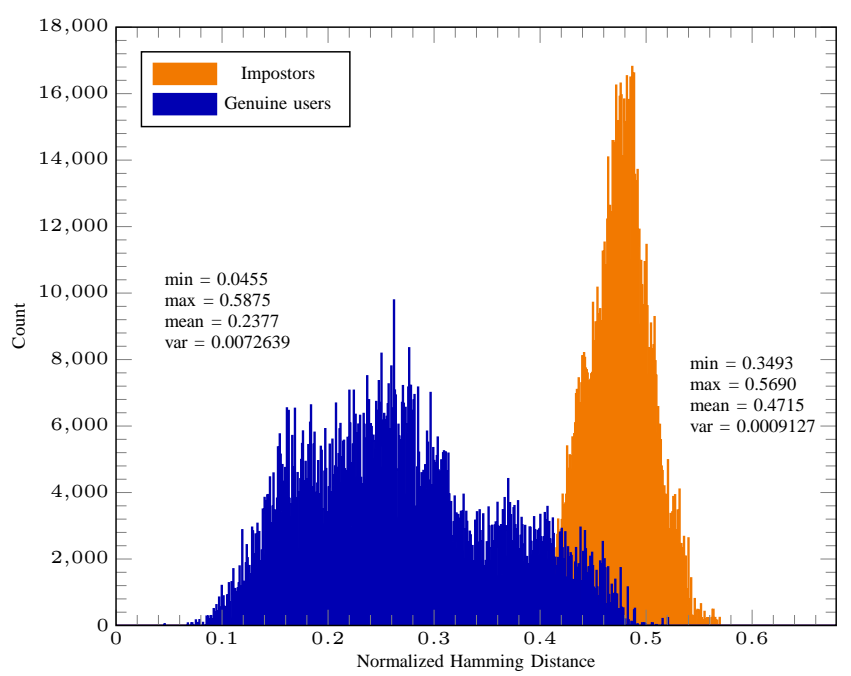

Fig. 5. Normalized Hamming distance for genuine users and impostors for Biosecure database using a single iris.

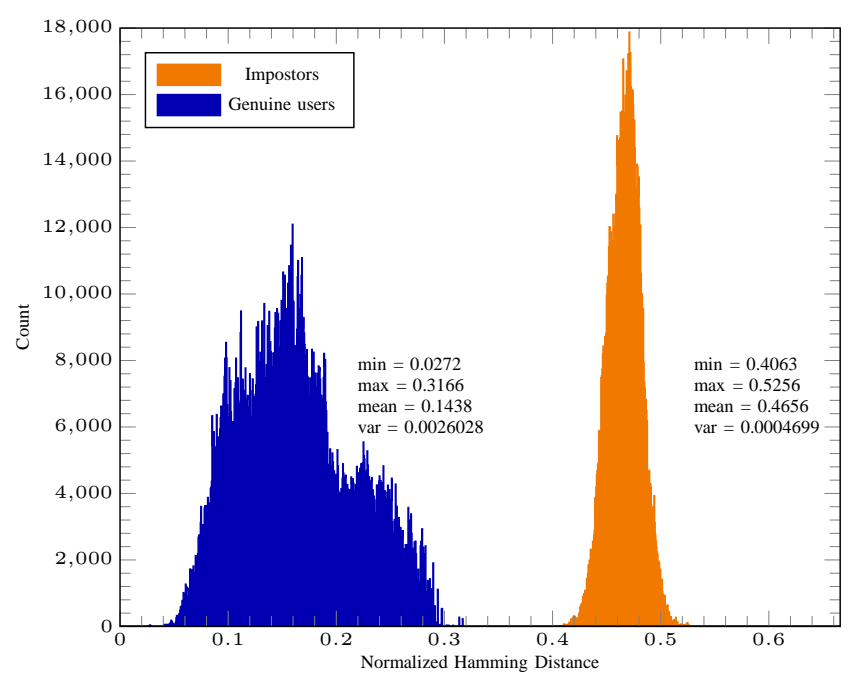

Fig. 6. Normalized Hamming distance for genuine users and impostors for Biosecure database using a single iris and employing shuffling key ( $\left.k_{\text {shuf }}\right)$, random numbers and rotation search.

\section{Performance of the proposed system for both irides}

For both irides, the block labelled iris code concatenation performs a concatenation of both left iris code and right iris code. Then a $\theta_{\text {ref }}$ sequence of 2,376 bits is sent to next step in the proposed system, illustrated in Figure 1. Some adjustments are performed for the system to perform as desired. Thus, the values $m=7$ and $k_{\text {shuf }}=396$ bits are employed, and we consider shortened RS codes of block length 61 over $\mathrm{GF}\left(2^{7}\right)$ and the Hadamard $(64,7,32)$ code. Values of $t_{\mathrm{RS}}$ greater than 22 are avoided because they increase the FAR, i.e., for $t_{\mathrm{RS}}>$ 22 the scheme begins to erroneously consider some impostors as genuine users.

Table IV exhibits results of experiments employing a single iris and two irides. Contrary to intuition, the processing time $t_{T}$ for experiments employing two irides is less than the corresponding processing time $t_{S}$ for a single iris. At beginning of our research we erroneously thought that $t_{T}$ would be greater than $t_{S}$ since the length of the iris code sequence for two irides is twice as long as that for a single iris. However it turns out that $t_{T}$ is almost twice less than $t_{S}$ for standard search and is almost five times less than $t_{S}$ when rotation search is employed. Analyzing all processes we observed that the processing time increases proportionally to the amount of errors which occur. When two irides are employed, we use a more powerful Hadamard code, i.e., the $(64,7,32)$ Hadamard code, and as a consequence the total number of decoding failures decreases, thus reducing the time spent to perform the corresponding experiments. If we take a look at the number of comparisons performed for each experiment, we can see that for two irides it is twice less than for a single iris.

TABLE IV

COMPARATIVE RESULTS OF EXPERIMENTS USING THE BIOSECURE DATA BASE FOR A SINGLE IRIS AND FOR TWO IRIDES $(\mathrm{C}++)$.

\begin{tabular}{|c|c|c|c|c|}
\hline & \multicolumn{2}{|c|}{ Single iris } & \multicolumn{2}{c|}{ Two irides } \\
\hline Search option & Standard & Rotation & Standard & Rotation \\
\hline Time (s) & 253 & 1,936 & 171 & 406 \\
\hline Comparisons & 132,000 & 132,000 & 66,000 & 66,000 \\
\hline Comparisons/s & 521 & 68 & 385 & 162 \\
\hline
\end{tabular}

Table $\mathrm{V}$ presents a percent FRR comparison between our proposed scheme, which is based on rotation search, and the scheme proposed in [4], which is based on standard search. As Table V shows, the use of rotation search achieved a better percent of FRR in all cases considered. Since in [4] the percent values of FRR only cover the range $10 \leq t_{\mathrm{RS}} \leq 14$, we have indicated by "N/A" (Not Available) the other values of $t_{\text {RS }}$. NIST-ICE data for a direct percent FRR comparison was available only when $t_{\mathrm{RS}}=10$. In this case our proposed scheme achieved a result 85 times better than that in [4]. However, for a comparison considering the percent of FRR and the number of $t_{\mathrm{RS}}$, our proposed scheme achieved a result about two times better for the NIST-ICE data base and three times better for the BIOSECURE and CASIA data bases. As a consequence it is now possible to recover cryptographic keys with 371 bits with measured FRR of $0.47 \%$ and FAR of $0 \%$ for NIST-ICE, employing both irides.

Figure 7 illustrates the situation where no user separation technique is employed and Figure 8 illustrates the user separation resulting from the application of a shuffling key $\left(k_{\text {shuf }}\right)$, random numbers and rotation search for both irides. 
TABLE V

PERCENT FRR FROM [4] AND ROTATION SEARCH FOR BOTH IRIDES. FAR IS ALWAYS ZERO FOR BOTH CASES. N/A = NOT AVAILABLE.

\begin{tabular}{|c|c|c|c|c|c|c|c|}
\hline & & \multicolumn{2}{|c|}{ BIOSECURE V1 } & \multicolumn{2}{c|}{ CASIA V2 } & \multicolumn{2}{c|}{ NIST-ICE } \\
\hline$t_{\mathrm{RS}}$ & $\|\mathbf{K}\|$ & {$[4]$} & Rotation & {$[4]$} & Rotation & {$[4]$} & Rotation \\
\hline 1 & 413 & N/A & $\mathbf{2 . 6 6 5}$ & N/A & $\mathbf{2 . 7 2 7}$ & N/A & $\mathbf{2 . 9 0 1}$ \\
\hline 2 & 399 & N/A & $\mathbf{1 . 7 4 8}$ & N/A & $\mathbf{0 . 9 1 3}$ & N/A & $\mathbf{1 . 5 1 4}$ \\
\hline 3 & 385 & N/A & $\mathbf{1 . 1 0 7}$ & N/A & $\mathbf{0 . 1 9 0}$ & N/A & $\mathbf{0 . 8 6 0}$ \\
\hline 4 & 371 & N/A & $\mathbf{0 . 6 5 4}$ & N/A & $\mathbf{0 . 0 2 9}$ & N/A & $\mathbf{0 . 4 7 1}$ \\
\hline 5 & 357 & N/A & $\mathbf{0 . 3 3 5}$ & N/A & $\mathbf{0}$ & N/A & $\mathbf{0 . 2 1 2}$ \\
\hline 6 & 343 & N/A & $\mathbf{0 . 1 4 8}$ & N/A & $\mathbf{0}$ & N/A & $\mathbf{0 . 0 9 8}$ \\
\hline 7 & 329 & N/A & $\mathbf{0 . 0 5 5}$ & N/A & $\mathbf{0}$ & N/A & $\mathbf{0 . 0 5 5}$ \\
\hline 8 & 315 & N/A & $\mathbf{0 . 0 0 9}$ & N/A & $\mathbf{0}$ & N/A & $\mathbf{0 . 0 2 9}$ \\
\hline 9 & 301 & N/A & $\mathbf{0 . 0 0 2}$ & N/A & $\mathbf{0}$ & N/A & $\mathbf{0 . 0 0 8}$ \\
\hline 10 & 287 & 1.03 & $\mathbf{0}$ & 0.67 & $\mathbf{0}$ & 0.34 & $\mathbf{0 . 0 0 4}$ \\
\hline 11 & 273 & 0.60 & $\mathbf{0}$ & 0.23 & $\mathbf{0}$ & 0.16 & $\mathbf{0}$ \\
\hline 12 & 259 & 0.17 & $\mathbf{0}$ & 0.13 & $\mathbf{0}$ & 0.11 & $\mathbf{0}$ \\
\hline 13 & 245 & 0.13 & $\mathbf{0}$ & 0.10 & $\mathbf{0}$ & 0.05 & $\mathbf{0}$ \\
\hline 14 & 231 & 0.10 & $\mathbf{0}$ & 0.07 & $\mathbf{0}$ & 0 & $\mathbf{0}$ \\
\hline 15 & 217 & N/A & $\mathbf{0}$ & N/A & $\mathbf{0}$ & N/A & $\mathbf{0}$ \\
\hline 16 & 203 & N/A & $\mathbf{0}$ & N/A & $\mathbf{0}$ & N/A & $\mathbf{0}$ \\
\hline 17 & 189 & N/A & $\mathbf{0}$ & N/A & $\mathbf{0}$ & N/A & $\mathbf{0}$ \\
\hline 18 & 175 & N/A & $\mathbf{0}$ & N/A & $\mathbf{0}$ & N/A & $\mathbf{0}$ \\
\hline 19 & 161 & N/A & $\mathbf{0}$ & N/A & $\mathbf{0}$ & N/A & $\mathbf{0}$ \\
\hline 20 & 147 & N/A & $\mathbf{0}$ & N/A & $\mathbf{0}$ & N/A & $\mathbf{0}$ \\
\hline 21 & 133 & N/A & $\mathbf{0}$ & N/A & $\mathbf{0}$ & N/A & $\mathbf{0}$ \\
\hline 22 & 119 & N/A & $\mathbf{0}$ & N/A & $\mathbf{0}$ & N/A & $\mathbf{0}$ \\
\hline
\end{tabular}

A comparison between Figure 7 and Figure 5, or between Figure 8 and Figure 6, indicates a considerable similarity. For either one iris or two irides the use of rotation search always gives results at least as good as those obtained with a standard search. This conclusion follows because rotation search comes into action only if the standard search fails to identify a user, and consequently rotation search employs more comparisons. However, the number of tests required for key regeneration and user identification when employing rotation search increases only by a factor of two on average in comparison to standard search, both for a single iris for two irides.

\section{CONCLUSIONS}

By employing rotated reference images of the iris we developed a rotation search that is far more efficient than the approach in [5] using a single iris for positive user identification and cryptographic key reconstruction. It is now possible to recover cryptographic keys with 198 bits with measured FRR of $0.24 \%$ and FAR of $0 \%$ for NIST-ICE-exp 1 employing a single iris. When two irides are employed, the rotation search is far more efficient than the approach in [4]. It is now possible to recover cryptographic keys with 371 bits with measured FRR of $0.47 \%$ and FAR of $0 \%$ for NIST-ICE. Table VI presents a comparison among systems proposed in the literature and three different instances of our proposed system.

The processing time for implementing rotation search remains smaller than the processing time for standard test employing MATLAB. For experiments employing two irides, we observed that the processing time required to perform an experiment is less than that required when a single iris is employed. The simulation results presented here are at least two times better than the best results achieved in the papers used for comparison, either for a single iris or for two irides.

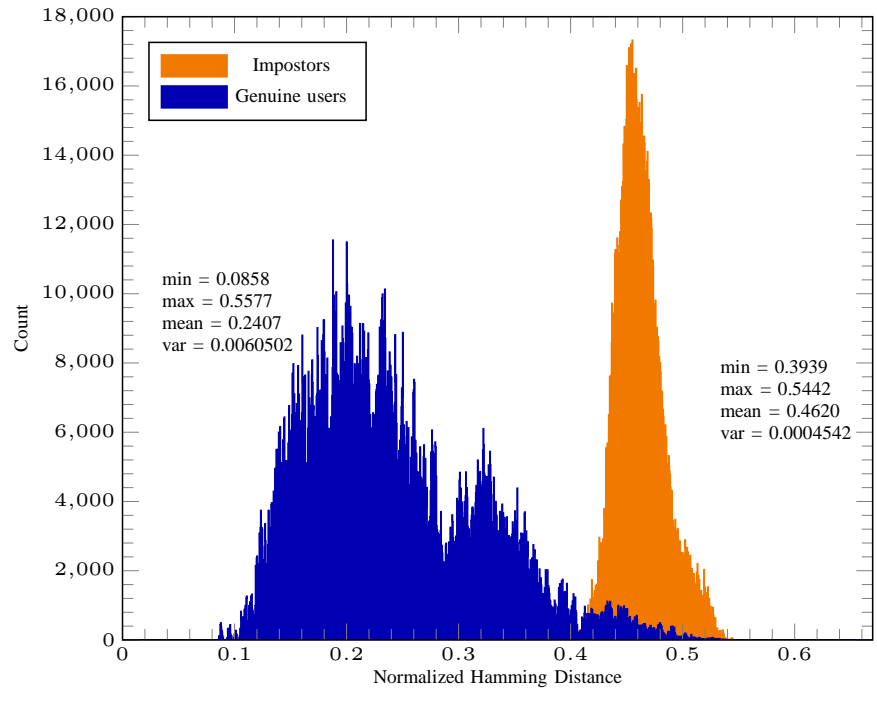

Fig. 7. Normalized Hamming distance for genuine users and impostors for Biosecure database using both iris.

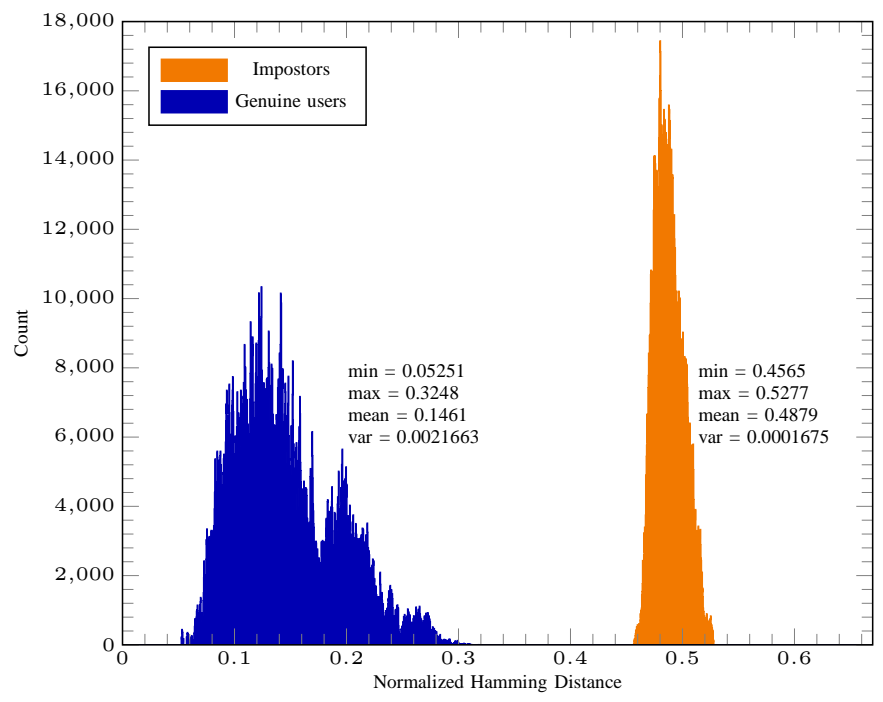

Fig. 8. Normalized Hamming distance for genuine users and impostors for Biosecure database using both iris and employing shuffling key $\left(k_{\text {shuf }}\right)$, random numbers and rotation search.

We notice that the rotation search can be implemented as an upgrade in most iris identification systems with some minor changes. Clearly, one can first perform a standard search, which can be followed by a rotation search if necessary. In other words, simulation results using rotation search are always at least as good as those based on a standard search. In the experiments performed with the data bases indicated earlier, rotation search always achieved the best results.

Finally, we remark that the rotation search test can be used in distinct biometric systems, which may employ other biometric features [19], [20]. We intend to investigate the efficiency of the rotation search test against other data bases, independent of the particular biometric feature, considering just that the feature vector is in binary form. We also intend to deepen our investigation of the behavior of different ECC techniques combined with rotation search and their effects 
TABLE VI

PERCENT FRR RESULTS FOR IRIS BIOMETRIC ALGORITHMS; (*) DENOTES MULTIBIOMETRIC SYSTEMS; ECC: ERROR-CORRECTING CODING; RSH:

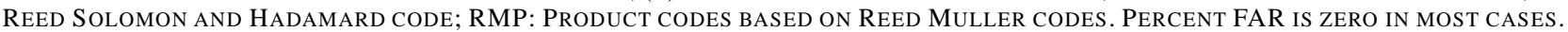

\begin{tabular}{|c|c|c|c|c|c|}
\hline Scheme & ECC & Key length (in bits) & FRR (\%) & FAR (\%) & Data base \\
\hline \hline Reference [3] & RS & 282 & 8.42 & 0 & NIST-ICE (right eye) \\
\hline Reference [5] & RS & $128 / 256$ & 0.76 & 0.10 & NIST-ICE (right eye) \\
\hline Reference [17] & RMP & 42 & 0.47 & 0 & NIST-ICE (right eye) \\
\hline Reference [18]* & RSH & 147 & 0.18 & 0 & NIST-ICE (both eyes) \\
\hline Reference [4]* $^{*}$ & RSH & 231 & 0 & 0 & NIST-ICE (both eyes) \\
\hline Reference [4]* $^{*}$ RSH & 287 & 0.34 & 0 & NIST-ICE (both eyes) \\
\hline Proposed $^{\text {RSH }}$ & $\mathbf{1 9 8}$ & $\mathbf{0 . 2 4}$ & $\mathbf{0}$ & NIST-ICE (right eye) \\
\hline Proposed* $^{*}$ & RSH & $\mathbf{2 7 3}$ & $\mathbf{0}$ & $\mathbf{0}$ & NIST-ICE (both eyes) \\
\hline Proposed* $^{*}$ & RSH & $\mathbf{3 7 1}$ & $\mathbf{0 . 4 7}$ & $\mathbf{0}$ & NIST-ICE (both eyes) \\
\hline
\end{tabular}

on the overall system security. In another scenario one may consider the investigation of the performance of combined biometric features [21], [22].

\section{ACKNOWLEDGEMENTS}

The first author is grateful to Dr. Danielle Camara, for introducing him to the field of biometrics, for supplying MATLAB simulation software for a single iris and for explaining details of the iris data bases. The second author acknowledges partial support of this work by the Brazilian National Council for Scientific and Technological Development (CNPq), Project No. 304696/2010-2.

\section{REFERENCES}

[1] G. N. Melo, V. C. da Rocha Jr. and J. S. Lemos-Neto, "User identification and key regeneration system employing rotated reference images of the iris," XXXIII Brazilian Telecommunications Symposium, Juiz de Fora-MG, Brazil, Sep. 2015, pp. 1-5.

[2] P. Tuyls, B. Skoric and T. Kevenaar, Security with Noisy Data: On Private Biometrics, Secure Key Storage and Anti-Counterfeiting, Springer, 2007.

[3] S. Kanade, D. P. B. A. Camara, E. Krichen, D. Petrovska-Delacrétaz and B. Dorizzi, "Three factor scheme for biometric-based cryptographic key regeneration using iris," The 6th Biometrics Symposium 2008 (BSYM2008), Tampa, Florida, USA, 2008, pp. 59-64. doi: 10.1109/BSYM.2008.4655523

[4] D. P. B. A. Camara, J. S. Lemos-Neto, and V. C. da Rocha Jr., "Multiinstance based cryptographic key regeneration system," JCIS, vol. 29, no. 1, May 2014. doi: 10.14209/jcis.2014.4

[5] S. Kanade, D. P. B. A. Camara, D. Petrovska-Delacrétaz and B. Dorizzi, "Application of biometrics to obtain high entropy cryptographic keys," Proceedings of World Academy of Science, Engineering and Technology, vol. 27, Hong Kong, China, March 2009, pp. 251-255.

[6] S. Barra, A. Casanova, F. Narducci and S. Ricciardi, "Ubiquitous iris recognition by means of mobile devices," Pattern Recognition Letters, vol. 57, pp. 66-73, 2015. doi: 10.1016/j.patrec.2014.10.011

[7] M. F. Zafar, Z. Zaheer and J. Khurshid, "Novel iris segmentation and recognition system for human identification," 10th International Bhurban Conference on Applied Sciences and Technology (IBCAST), Islamabad, 2013, pp. 128-131.

[8] H. Betaouaf and A. Bessaid, "A biometric identification algorithm based on retinal blood vessels segmentation using watershed transformation," 8th International Workshop on Systems, Signal Processing and their Applications (WoSSPA), Algiers, Algeria, May 2013, pp. 256-261. doi: 10.1109/WoSSPA.2013.6602372

[9] C. Narmatha and S. Manimurugan, "A new approach for iris image identification using modified contour segmentation," IEEE International Conference on Green Computing Communication and Electrical Engineering (ICGCCEE), Coimbatore, India, March 2014, pp. 1-7. doi: 10.1109/ICGCCEE.2014.6921399

[10] A. Mallikarjuna and S. Madhuri, "Biometric security techniques for IRIS recognition system," IJRCCT, vol. 2, no. 8, pp. 589-593, 2013.
[11] J. Bringer, C. Morel and C. Rathgeb, "Security analysis of Bloom filterbased iris biometric template protection," International Conference on Biometrics (ICB), Phuket, 2015, pp. 527-534.

[12] J. Bringer, H. Chabanne and C. Morel, "Shuffling is not sufficient: Security analysis of cancelable iris codes based on a secret permutation," IEEE International Joint Conference on Biometrics (IJCB), Clearwater, FL, 2014, pp. 1-8.

[13] K. Nandakumar and A. K. Jain, "Biometric template protection: bridging the performance gap between theory and practice," IEEE Signal Processing Magazine, vol. 32, no. 5, pp. 88-100, Sept. 2015. doi: 10.1109/MSP.2015.2427849.

[14] "BioSecure Network of Excellence", Avaible: http://www.biosecure.info. Accessed Mar. 21, 2016

[15] National Institute of Science and Technology (NIST), "Iris Challenge Evaluation," 2005, Avaible: http://iris.nist.gov/itl/iad/ice.cfm. Accessed Mar. 21, 2016

[16] F. J. MacWilliams and N. J. A. Sloane, The Theory of Error-Correcting Codes, North Holland, 1988.

[17] J. Bringer, H. Chabanne, G. Cohen, B. Kindarji and G. Zmor, "Theoretical and practical boundaries of binary secure sketches," IEEE Transactions on Information Forensics and Security, vol. 3, no. 4, pp. 673-683, 2008. doi: 10.1109/TIFS.2008.2002937.

[18] S. Kanade, D. Petrovska-Delacretaz, and B. Dorizzi,"Multi-biometrics based cryptographic key regeneration scheme," IEEE 3rd International Conference on Biometrics: Theory, Applications and Systems, Washington DC, USA, 2009, pp. 1-7. doi: 10.1109/BTAS.2009.5339034

[19] Maltoni, D., Maio, D., Jain, A. and Prabhakar, S., Handbook of Fingerprint Recognition, Springer, 2009.

[20] J. M. Guo, Y. F. Liu, M. H. Chu, C. C. Wu and T. N. Le, "Contact-free hand geometry identification system," 18th IEEE International Conference on Image Processing (ICIP), Brussels, 2011, pp. 3185-3188.

[21] Z. Wang, C. Liu, T. Shi and Q. Ding. "Face-palm identification system on feature level fusion based on CCA," J. Inform. Hiding Multimedia Signal Processing, vol. 4, pp. 272-279, 2013.

[22] S. Kanade, D. Petrovska-Delacretaz and B. Dorizzi,"Obtaining cryptographic keys using feature level fusion of iris and face biometrics for secure user authentication," IEEE Computer Vision and Pattern Recognition Workshops (CVPRW), San Francisco, CA, USA, June 2010, pp. 138-145. doi: 10.1109/CVPRW.2010.5544618

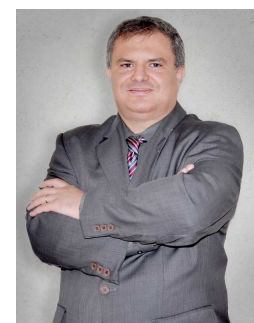

Guilherme Nunes Melo was born in Recife, Pernambuco, Brazil, on august 11,1975 . He received the B.Sc. (1998) and the M.Sc.(2010) degrees in Electrical/Electronics Engineering from the Federal University of Pernambuco, Recife, Brazil. He is currently a doctoral student with the Communications Research Group, Department of Electronics and Systems, Federal University of Pernambuco. He joined in the Brazilian Telecommunications Society in 2015. His professional experience includes research and teaching. Mr. Melo's research interests are in applied digital information theory, error-correcting codes, digital communications, digital signal processing, micro controllers and embedded systems. 


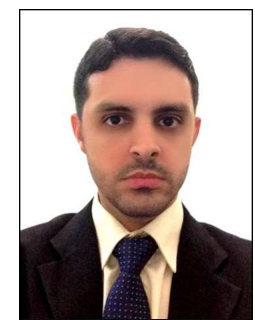

José Sampaio de Lemos Neto was born in Bezerros, Pernambuco, Brazil, on November 27, 1980. He received the B.Sc. (2004), M.Sc. (2011) and D.Sc. (2015) degrees, all in Electrical/Electronics Engineering from the Federal University of Pernambuco, Recife, Brazil. In 2015 he joined the faculty of the Federal University of Pernambuco, Recife, Brazil, as an Associate Professor. He was a research assistant in the project "Cryptographic Security based on Noisy Physical Elements" developed by Dr. D. P.

B. A. Camara and supervised by Prof. V. C. da Rocha, Jr.. He joined in the Brazilian Telecommunications Society in 2010. His professional experience includes research and teaching. Mr. LemosNeto's research interests are in applied digital information theory, errorcorrecting codes, digital communications, digital signal processing and applied mathematics.

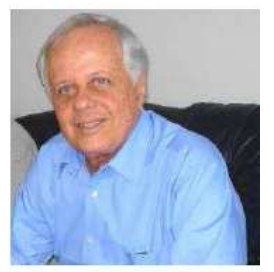

Valdemar C. da Rocha Jr. (M77, SM04, LSM13) was born in Jaboatão, Pernambuco, Brazil, on August 27, 1947. He received in 1970 the B.Sc. degree in Electrical/Electronics Engineering from the Escola Politécnica, Recife, Brazil, and in 1976 he received the Ph.D. degree in Electronics from the University of Kent at Canterbury, U.K. He joined the faculty of the Federal University of Pernambuco, Recife, Brazil, in 1976 as an Associate Professor and founded its Electrical Engineering Postgraduate Programme. He served as Department Chair (19921996), and in 1993 he became Professor of Telecommunications. He was editor for Coding Theory and Techniques, Journal of Communication and Information Systems, co-sponsored by the Brazilian Telecommunications Society and the IEEE Communications Society, and has been a reviewer for a number of scientific journals including IET Electronics Letters, IET Communications and IEEE Transactions on Information Theory. He has also been involved in the organization of conferences in Brazil and abroad. He is a founder (2002) and past President (2002-2004) of the IEEE Information Theory Society Chapter, Brazil Council. He is founder (2003) and VicePresident for three consecutive terms (2003-2015) of the Institute for Advanced Studies in Communications. He is a founding member (1983) of the Brazilian Telecommunications Society, served as Vice-President for two terms (2000-2004) and as President also for two terms (2004-2008). He joined the IEEE Communications Society in 1977 and the IEEE Information Theory Society in 1981. He is a Member (1982) of the Brazilian Society of Applied and Computational Mathematics, and a Fellow (1992) of the Institute of Mathematics and its Applications, UK. During 1990-1992, he was a Visiting Professor at the Swiss Federal Institute of Technology-Zurich, Institute for Signal and Information Processing. In 2005-2006 he was a Visiting Professor at the Institute of Integrated Information Systems, University of Leeds, UK, and in 2007 he was a Visiting Professor at the Department of Communication Systems, Lancaster University, UK. Prof. da Rocha research interests are in applied digital information theory, including error-correcting codes and cryptography. He has published over 100 engineering and scientific papers, including journal and conference papers, and the books Communication Systems, Springer, 2005, and Elements of Algebraic Coding Systems, Momentum Press, 2014. He is currently a Member of the IEEE Alexander Graham Bell Medal Committee. 